\title{
Numerical Heliospheric Simulations as Assisting Tool for Interpretation of Observations by STEREO Heliospheric Imagers
}

\author{
Dusan Odstrcil • Victor J. Pizzo
}

Received: 10 December 2008 / Accepted: 31 August 2009 / Published online: 13 October 2009

(C) The Author(s) 2009. This article is published with open access at Springerlink.com

\begin{abstract}
The interpretation of multi-spacecraft heliospheric observations and threedimensional reconstruction of structured and evolving solar wind is challenging. This is especially true for the interpretation of white-light structures observed by the Heliospheric Imagers (HI) onboard STEREO spacecraft since their appearance depends on three-dimensional geometric factors. Numerical simulations can provide global context and suggest what may and may not be observed. We use the heliospheric code ENLIL to simulate various scenarios of well-defined corotating solar wind streams and ejected transient density structures, and we generate from the solutions synthetic white-light images at various locations. We illustrate that corotating interaction regions (CIRs) show up differently in HI-2A and HI-2B and that they may appear as transient structures in HI-2A but not in HI-2B. This asymmetry is caused by differing Thomson scattering responses. Further, we illustrate that a given interplanetary coronal mass ejection (ICME) may exhibit drastically different white-light brightness depending on the observing position and that some ICMEs can eventually reach Earth without being detected by the imagers. Finally, we demonstrate application of the modeling system through simulation of the 24-25 January 2007, 31 December 2007 and 26 April 2008 CMEs.
\end{abstract}

Keywords Coronal mass ejection · Solar wind · Numerical simulation · STEREO Heliospheric Imager

STEREO Science Results at Solar Minimum

Guest Editors: Eric R. Christian, Michael L. Kaiser, Therese A. Kucera, O.C. St. Cyr

D. Odstrcil

Cooperative Institute for Research in Environmental Sciences, University of Colorado, 325 Broadway, Boulder, CO 80305, USA

D. Odstrcil $(\varangle)$. V.J. Pizzo

NOAA/Space Weather Prediction Center, 325 Broadway, Boulder, CO 80305, USA

e-mail: dusan.odstrcil@noaa.gov 


\section{Introduction}

The Heliospheric Imagers (HI) onboard STEREO A and B spacecraft provide images of the solar corona from two vantage points in the ecliptic plane near $1 \mathrm{AU}$. The HI-2s map the inner heliosphere with two-hour cadence and high sensitivity. The two STEREO spacecraft are continuously separating from Earth and each other in opposite directions, thus providing different perspectives on corotating and transient heliospheric structures. Therefore, it is extremely important to understand how to interpret three-dimensional structures that can be obtained at each phase of the STEREO orbit. Because the coronal mass ejections (CMEs) are visible due to Thomson scattering, the intensity of an observed CME is dependent on the distance from the Sun and on the angle it makes with the observed plane-of-the-sky. Thus, the interpretation of HI observations is not straightforward, and in contrast to coronagraph observations, depends upon geometrical factors.

To understand the visibility of ICMEs and the ability to discern the three-dimensional structure we developed tools which simulate corotating interaction regions (CIRs) and/or CMEs and provide visualization output directly comparable with the observations. We first compute the background solar wind with our three-dimensional magnetohydrodynamic (MHD) code which uses for input the WSA (Wang-Sheeley-Arge) empirical coronal model output. Then we launch hydrodynamic ejecta into the heliospheric computational domain to simulate CMEs. Finally, we use the simulated distribution of the solar wind (SW) density to generate synthetic images of the total white-light brightness as might be observed from STEREO spacecraft.

Modeling the origin of CMEs is still in the research phase and it is not expected that real events can be routinely simulated in the near future. Therefore, we developed an intermediate modeling system which uses inputs derived from coronagraph observations to launch three-dimensional ejecta into the background solar wind. This modeling system is fast and robust, and it enables simulation of virtually any observed CME event in the inner- and midheliospheres. Therefore, a combination of a numerical MHD model for the heliosphere and one or more semi-analytic models for near-Sun disturbances represents a much needed practical approach. This "hybrid" modeling scheme ignores specifics of the magnetic eruption process; it takes the observed resulting structure and launches it into the heliosphere where it evolves as it interacts with the background SW. A combination of coronagraph fittings using the cone model and three-dimensional numerical computations were applied to real events (Odstrcil, Riley, and Zhao, 2004; Odstrcil, Pizzo, and Arge, 2005). Although empirical cone models (e.g., Zhao, Plunkett, and Liu, 2002; Michalek, Gopalswamy, and Yashiro, 2003; Xie, Ofman, and Lawrence, 2004) offer no information about the important internal magnetic structure of a CME, they do provide observationally based model input that is readily determined.

In this article, we start with hypothetic scenarios to provide basic insight into complex three-dimensional phenomena. We simulate simple CIRs and CMEs and illustrate how they might be seen at different projected heliocentric distances from different vantage points. Then, we use the modeling tool to simulate the 24-25 January 2007, 31 December 2007 and 26 April 2008 ICMEs. These events include interactions of two ICMEs with each other and with the corotating streamer belt. Their interpretation benefits from comparison of observational and numerical results.

\section{Modeling System}

We developed ENLIL, the three-dimensional MHD numerical code, to simulate corotating and transient SW disturbances in the inner- and mid-heliospheres (Odstrcil, Dryer, and 
Smith, 1996; Odstrcil and Pizzo, 1999a, 1999b). ENLIL provides values of all MHD quantities (density, temperature, and velocity and magnetic field components) at any location and time within the computational domain (typically between 0.1 and 1.7 AU to include Mars). An important feature of ENLIL is its ability to solve two additional continuity equations used for tracing the injected CME material and the IMF polarity (Odstrcil and Pizzo, 1999a, $1999 b)$. These quantities have been helpful in three-dimensional visualization and analysis of numerical results (e.g., Odstrcil, 2003). ENLIL is driven by time-dependent values of all MHD quantities at the inner boundary of its heliospheric computational domain (see the next two sections). There are two steps in the specification of boundary values: (1) specify corotating values for the background SW and (2) superimpose time-dependent values for launching one or more transient disturbances. The inner boundary of the computational domain was chosen to lie in the "super-critical" flow region (where the bulk SW speed exceeds the fast-mode MHD speed; usually at $21.5 \mathrm{Rs}$ ) which simplifies the numerical solution.

ENLIL produces three-dimensional distributions of the solar wind density within the computational domain with a cadence of two hours. These data are used to generate synthetic images of the total brightness. An integration of the Thomson scattering formulae is performed along the line-of-sight of the observer through the corona from a given observer's location at a specified spatial resolution and field-of-view (FOV). The white-light corona and heliosphere are optically thin, so that the appearance on an image is the sum of contributions all along the line-of-sight. Thus, the appearance of a structure is highly dependent on its location with respect to the so-called Thomson surface, where the scattering response is greatest (Vourlidas and Howard, 2006).

\section{Corotating Interactions Regions}

Accurate computation of the background SW parameters is crucial for predicting co-rotating stream structures and for transient disturbances (interplanetary shocks and CMEs) that propagate and interact with those background structures. Currently, ENLIL can utilize output files produced by two different coronal models: (1) the three-dimensional MHD coronal MAS (Magnetohydrodynamics Around Sphere) model (developed at SAIC; see Riley, Linker, and Mikic, 2001) that uses synoptic maps of the photospheric magnetic field provided by the Kitt Peak National Observatory and (2) the empirical WSA model (Arge and Pizzo, 2000; Arge et al., 2003) that uses data from the Mount Wilson Solar Observatory, Wilcox Solar Observatory, and NSO/GONG network. For prediction runs we use WSA because it runs much faster than real time and because it shows very good validation results (Owens et al. 2005, 2008), therefore the WSA model outputs are used in this article. Validation of the background SW predictions by the WSA-ENLIL is performed at NSF/CISM (Owens et al., 2008) and NASA/CCMC (Lee et al., 2008) and we anticipate further improvements in prediction accuracy.

We simulate the tilted streamer belt similar to that used by Odstrcil and Pizzo (1999a, $1999 \mathrm{~b}$ ). The dense stream is $20^{\circ}$ wide, and it is inclined $30^{\circ}$ to the equatorial plane. Numerical simulations were conducted up to $2.1 \mathrm{AU}$, but we display results up to $1.1 \mathrm{AU}$. Figure 1 shows the corotating heliospheric stream structure in the equatorial plane at two different times separated by seven days. It is assumed that the STEREO spacecraft are $45^{\circ}$ away from the Earth. Thus, both spacecraft are immersed in the same stream structure. The left panel shows the STEREO B (blue color) just inside the density enhancement and its FOV is directed ahead of it along its orbit. Note that the tilted streamer belt crosses the Equator at the STEREO B location and it stretches on southward, extending about $30^{\circ}$ below the 


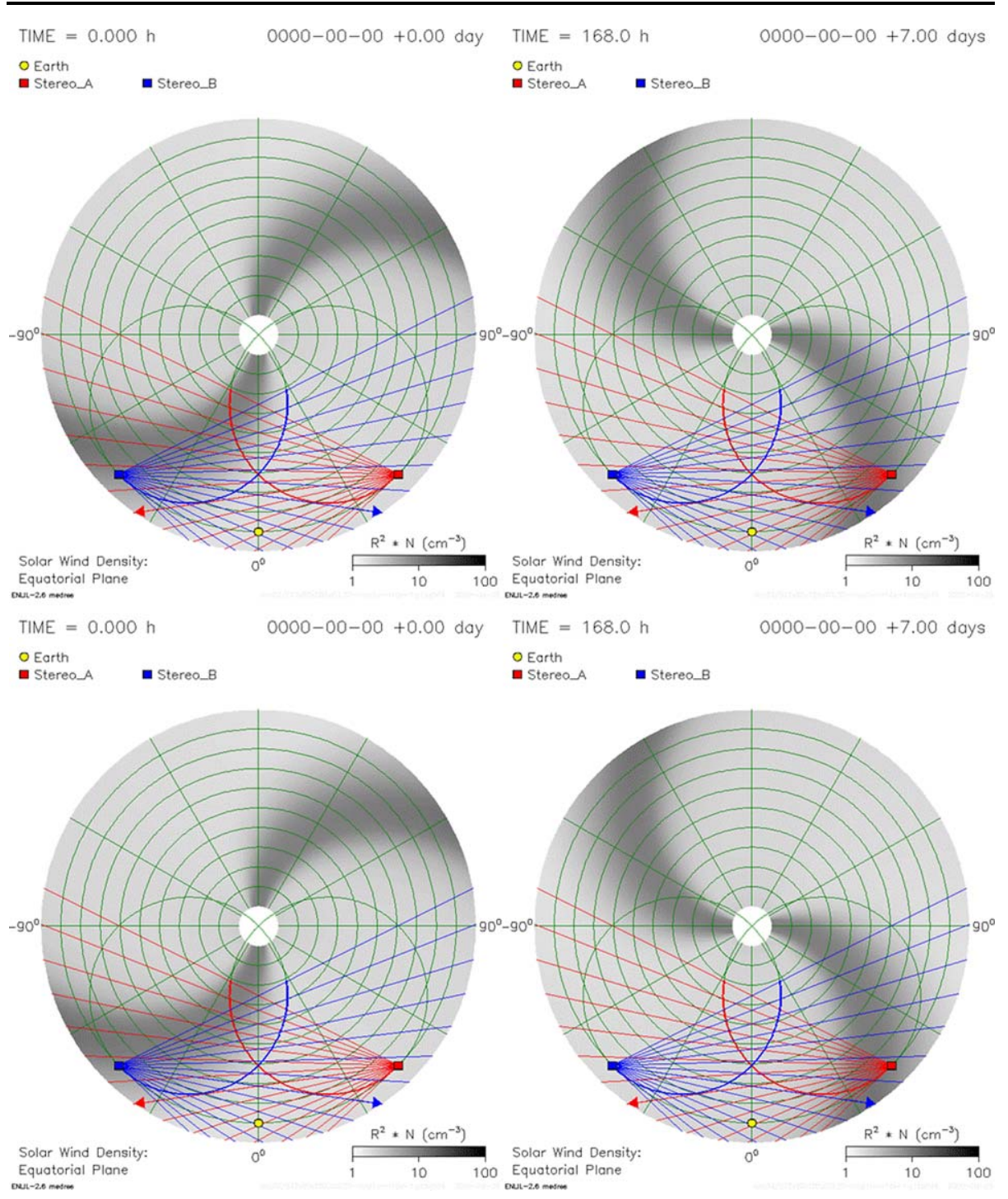

Figure 1 Simulated solar wind density, scaled by $\left(r / R_{\mathrm{AU}}\right)^{2}$, shown between 0.1 and $1.1 \mathrm{AU}$ on the equatorial slice passing through Earth (viewed from the North). The top (bottom) row shows results for the declining (rising) streamer belt at different times. The grey scale is given at the bottom. These panels also show positions of STEREO spacecraft with respective field-of-views (light red and blue lines separated by $5^{\circ}$ ) and corresponding Thomson curves (thick red and blue curves) marking the peak in the white-light scattering toward the observer. Heliocentric distance is indicated by green concentric rings separated by $0.1 \mathrm{AU}$.

equatorial plane and crossing over the STEREO A spacecraft. The right panel shows that STEREO A (red color) is immersed in the same stream structure seven days later, but its FOV is directed behind it, facing into the trailing stream rarefaction. Note that the tilted streamer belt crosses the Equator at the STEREO A location, stretching northward and about $30^{\circ}$ above the equatorial plane at the STEREO B location.

Figure 2 shows the corresponding synthetic white-light images as they might be observed by HI-2A (top row) and HI-2B (bottom row), located at positions indicated in Figure 1. The 


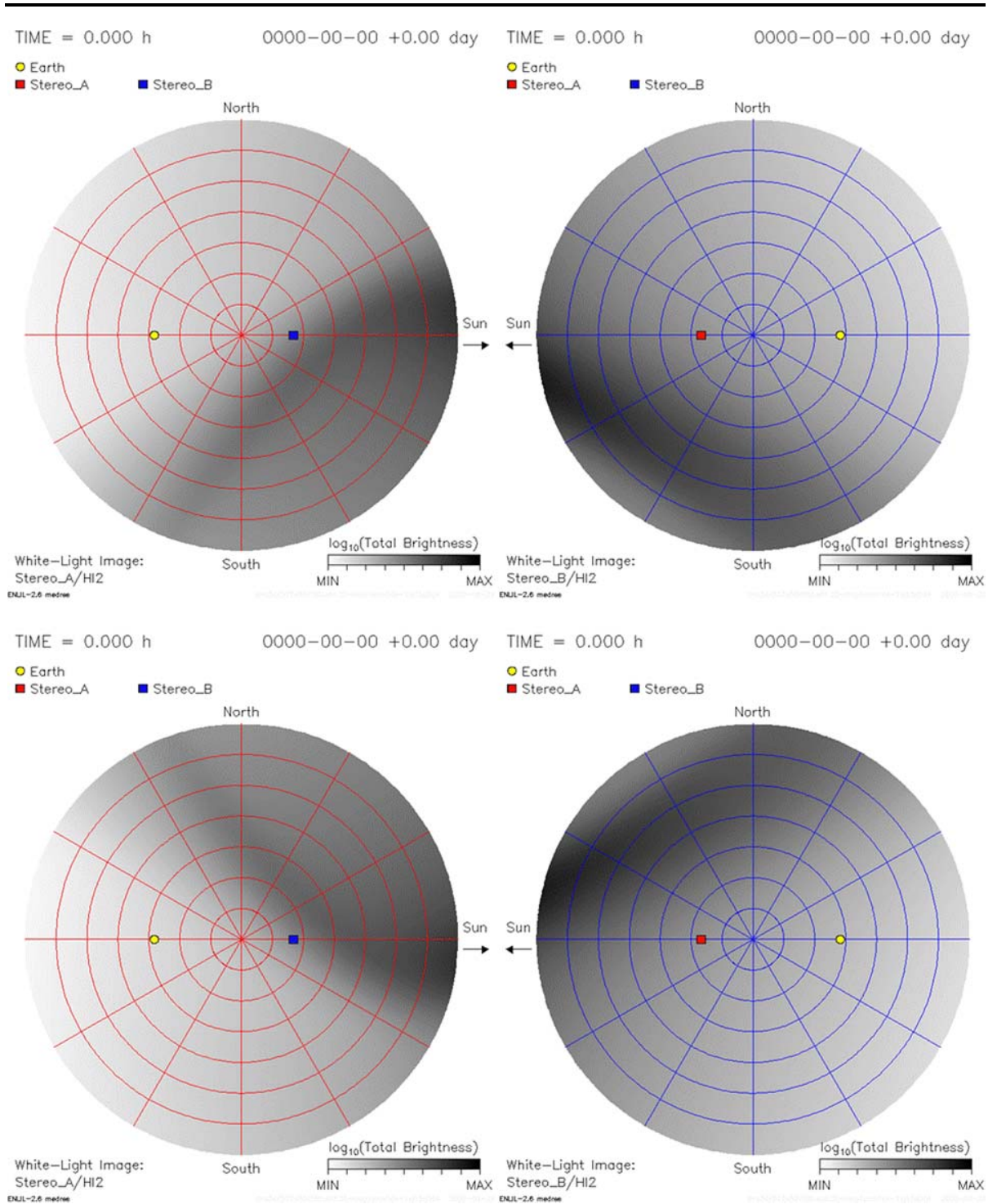

Figure 2 Synthetic white-light images as might be seen by STEREO HI-2 instruments. Four panels show images corresponding to numerical results presented in Figure 1. The top (bottom) row shows images for the declining (rising) streamer belt for STEREO HI-2A (2B) in the left (right) column. The total brightness is shown using the color scale given at the bottom of the each panel. Concentric circles correspond to the view directions displayed in Figure 1, and they are separated by $5^{\circ}$.

heliospheric streamer belt appears at the dark structure. It is highly inclined when the dense streamer crosses the Thompson surface and it is flat and occupies the upper (and, lower after 14 days) part of the FOV. Note that the streamer belt is inclined by $30^{\circ}$ and the maximum elongation of the HI- $2 \mathrm{~s}^{\prime} \mathrm{FOV}$ is $35^{\circ}$. Thus, the simulated dense streamer belt goes up and down across the whole FOV. 


\section{Interplanetary Coronal Mass Ejections}

We develop a procedure that generates time-dependent boundary conditions by launching a hydrodynamic plasma cloud through the inner boundary of the heliospheric computational domain into the background SW (Odstrcil and Pizzo, 1999a, 1999b). Parameters describing the plasma cloud, such as the location (latitude and longitude), speed, and angular width, can be obtained by empirical techniques using the so-called cone model (Zhao, Plunkett, and Liu, 2002; Michalek, Gopalswamy, and Yashiro, 2003; Xie, Ofman, and Lawrence, 2004) that fit observations of CMEs. The three-dimensional distribution of the density and temperature has to be specified for completeness; for simplicity, we usually assume a homogeneous spherical structure. We solve an additional equation to trace the evolution of the plasma cloud. Launching a spherical, over-pressured cloud into the heliospheric code can simulate transient heliospheric disturbances (Odstrcil, Riley, and Zhao, 2004; Odstrcil, Pizzo, and Arge, 2005). This models the global context of ICMEs with respect to corotating SW structures and it enables prediction of whether a shock and/or ejecta will hit or miss geospace. Density enhancement is a free parameter. This approach is numerically robust, and although simple, it is believed to be more accurate than empirical time-of-arrival formulae (e.g., Gopalswamy et al., 2001; Schwenn et al., 2005) due to simulation of realistic background SW and three-dimensional dynamic effects. Taktakishvili et al. (2009) analyzed the performance of this modeling system implemented at NASA/CCMC and confirmed its capability to predict the arrival times of ICMEs more accurately than the existing empirical models. Further analysis investigated the effect of the background SW on the ICME propagation (Case et al., 2008) and sensitivity of relative locations of the ICME and streamer belt (Lee et al., 2008).

We launch an over-pressured hydrodynamic structure similar to that used by Odstrcil and Pizzo (1999a, 1999b) into an assumed uniform background solar wind velocity of $450 \mathrm{~km} \mathrm{~s}^{-1}$ at the inner heliospheric boundary. The injected plasma cloud is initially a homogeneous spherical structure, with a speed of $900 \mathrm{~km} \mathrm{~s}^{-1}$. It has the same temperature as the background solar wind but six times larger plasma density. We consider two ejecta with angular widths of $20^{\circ}$ and $90^{\circ}$.

Figure 3 shows the two ICMEs, one narrow (top row) and one broad (bottom row), as might be viewed from two vantage points. As the narrow ICME propagates outward, its diameter slightly expands since the ICME is initially set-up as an over-pressured structure. The broader ICME behaves very similarly. Due to angular expansion, dynamic compression, and interaction with the homogeneous background solar wind (dark area indicates high density region), both ICMEs acquire characteristic arc shapes followed by large trailing rarefactions (white area indicates low density region).

Figure 4 shows the corresponding synthetic images. Due to the symmetry of the configurations, we present images for HI-2A only. It can be seen from Figure 3 that, in the case of small spacecraft separation, there is a "hole" through which narrow, Earth-directed ICMEs can propagate without being detected by STEREO HI-2s' instruments. They may eventually be detected just before their arrival at Earth when they encounter regions more favorable for scattering the white-light toward the observer. As the spacecraft separation increases, Earthdirected ICMEs are reliably detected. Note that when the ICME leading edge is parallel to the Thomson curve, the structure shows up as a very broad structure. On the other hand, when the ICME leading edge is perpendicular to the Thomson curve, the structure is more visible and appears as a relatively thin structure. Our simulated case is favorable for being detected by HI-2s since the curved leading edge of the ICME causes the line-of-sight to traverse through a lengthy column of the compressed density (see Figures 3 and 4). 


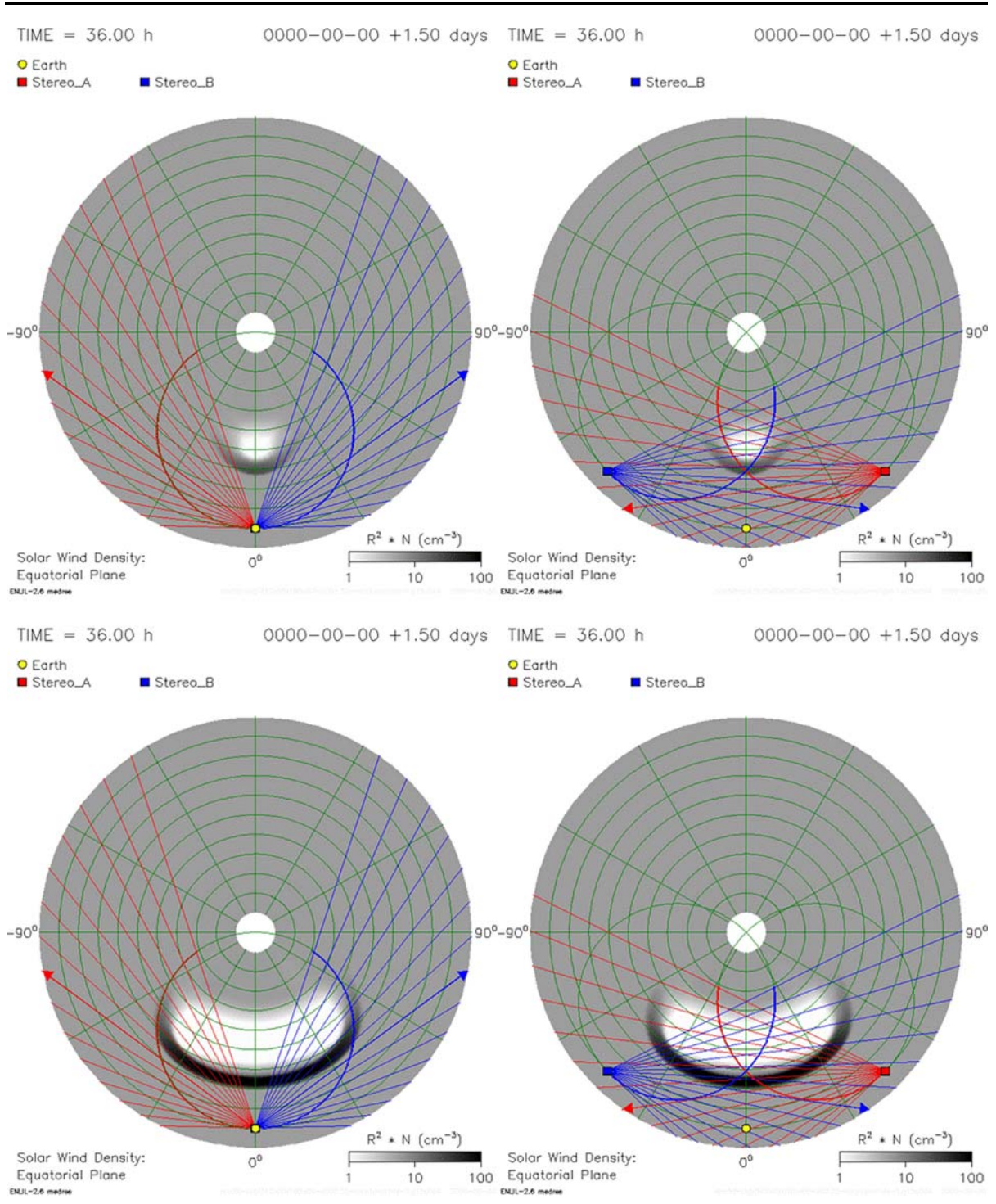

Figure 3 Numerical simulation of narrow and broad ICMEs. The top row shows the ICME with the original angular width of $20^{\circ}$ together with STEREO A and B spacecraft separated by $0^{\circ}$ (left) and $90^{\circ}$ (right). The bottom row shows the ICME with the original angular width of $90^{\circ}$ together with STEREO A and B spacecraft separated by $0^{\circ}$ (left) and $90^{\circ}$ (right). Quantities are shown as described in Figure 1.

\section{Simulation of Real Events}

The ability to determine the three-dimensional structure of the corotating and transient heliospheric disturbances is of primary importance for effective space weather research and forecasting. The use of iterative numerical simulations and well-considered visualization tools are crucial in this effort. We apply our modeling system to select well-observed ICMEs in order to understand the global context, provide modeling support to remote and in-situ observations, and obtain feedback for further improvements of the model. 


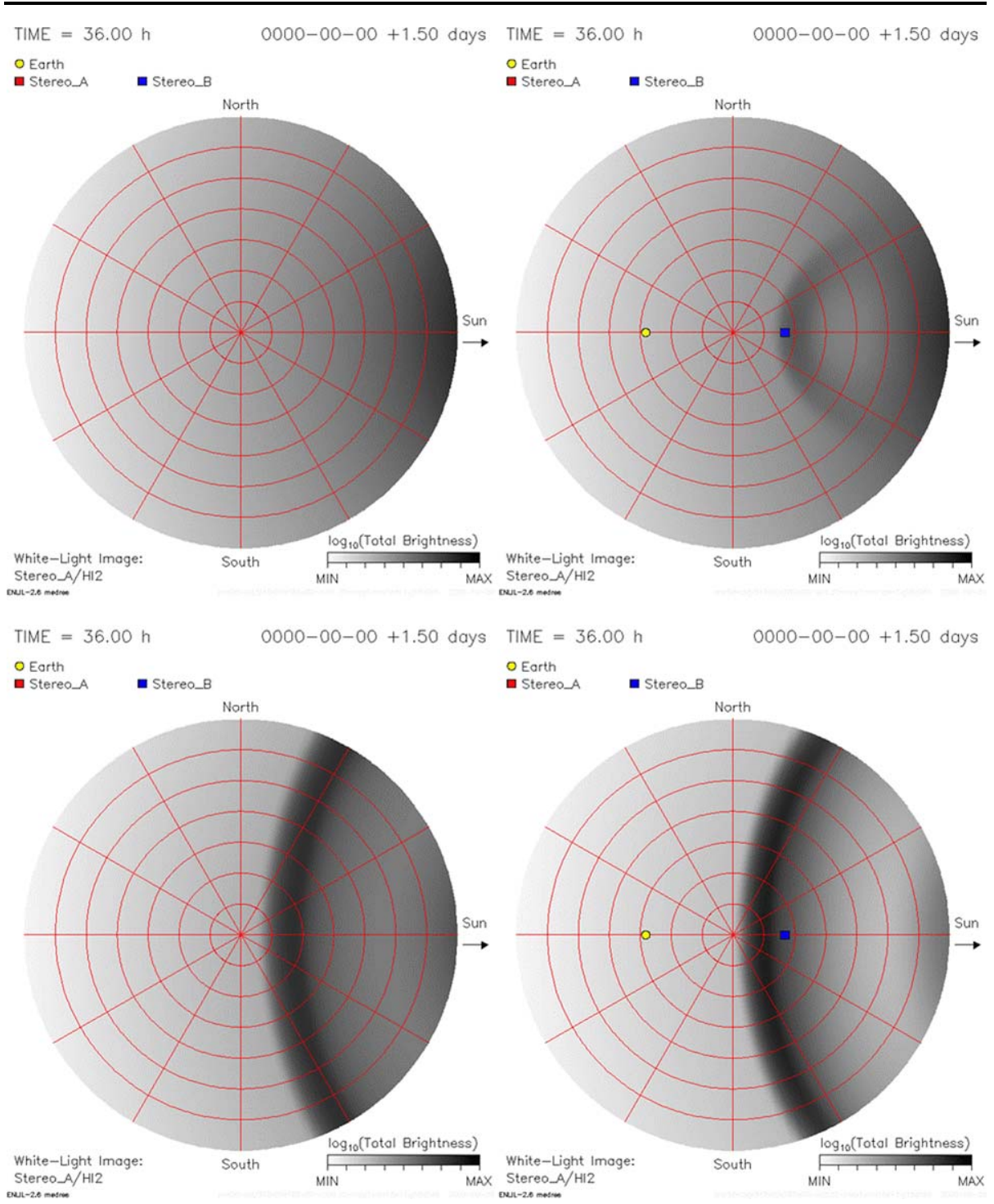

Figure 4 Synthetic white-light STEREO HI-2A images corresponding to the ICME parameters and times displayed in Figure 3. Quantities are shown as described in Figure 2.

Heliospheric disturbances caused by the 24 and 25 January 2007 east-limb CMEs were among the very first observed by STEREO HI-2s (Harrison et al., 2008). They were also observed by the SMEI all-sky heliospheric imager (Webb et al., 2009). Modeling these heliospheric disturbances can serve to illustrate our tool. There were no in-situ observations of ICMEs, thus all interpretation of the heliospheric disturbances depend on the remote observations only. Figures 5 and 6 present the composite display we use for understanding the global context. It combines simulated solar wind density with synthetic white-light images, as described in previous sections. The figures show density structures caused by CIRs and 


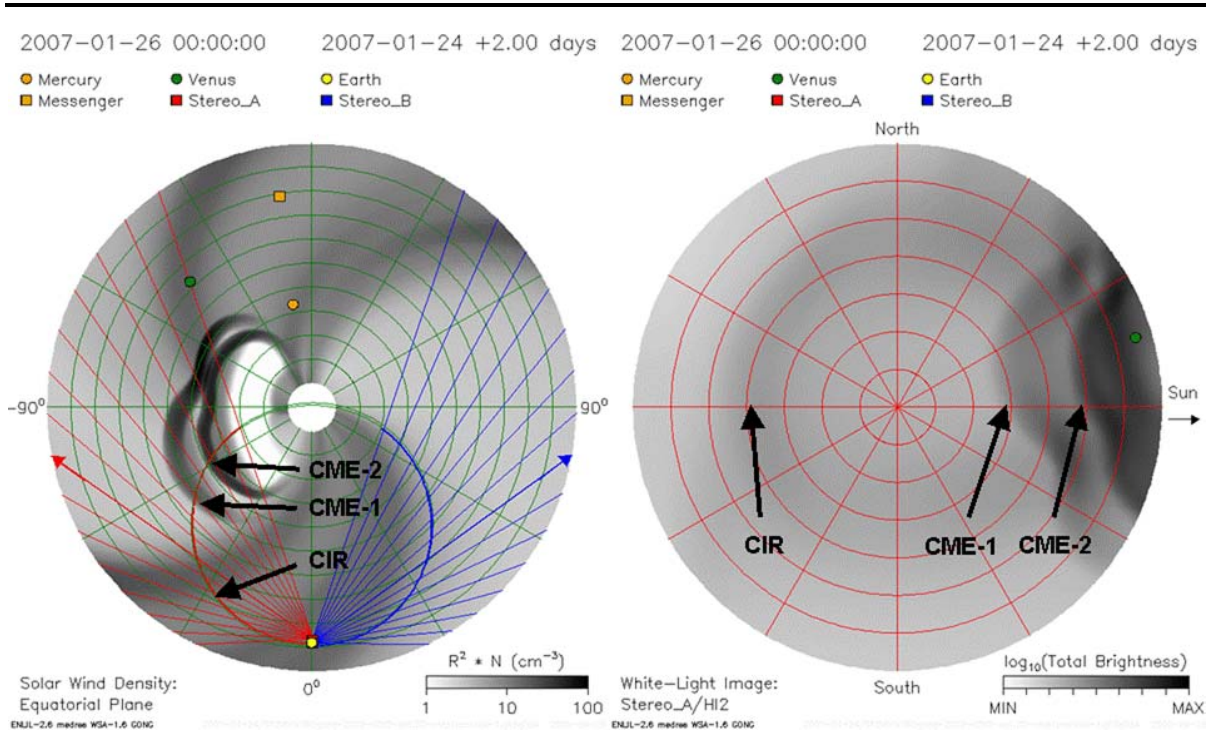

Figure 5 Results of heliospheric simulations of 24-25 January 2007 ICMEs are shown between 0.1 and 1.1 AU on 26 January 2007 00:00 UT. Left panel shows the solar wind plasma density, scaled by $\left(r / R_{\mathrm{AU}}\right)^{2}$, on the equatorial slice passing through Earth. The color scale is given at the bottom. Planets and spacecraft are marked by symbols given at the left. This panel also shows positions of STEREO spacecraft with respective field-of-views (light red and blue lines; axes have arrows indicating the viewing direction) and corresponding Thomson curves (thick red and blue curves) marking the peak in the white-light scattering toward the observer. Right panel shows synthetic white-light images as might be seen by STEREO HI-2A instruments. The total brightness is shown using the color scale given at the bottom. This panel also shows concentric circles that correspond to the view directions displayed in the left panel as lines.

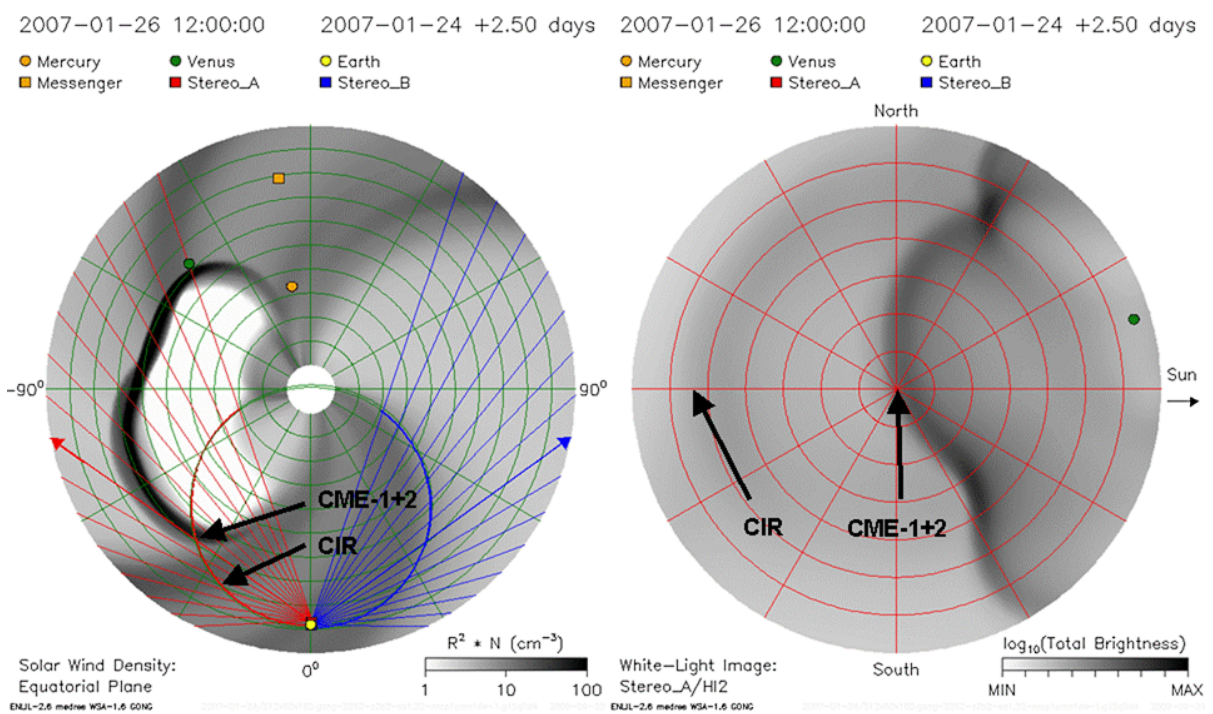

Figure 6 The same as in Figure 5 but results are shown on 26 January 2007 12:00 UT. At that time the faster ICME has overtaken the preceding slower one. 


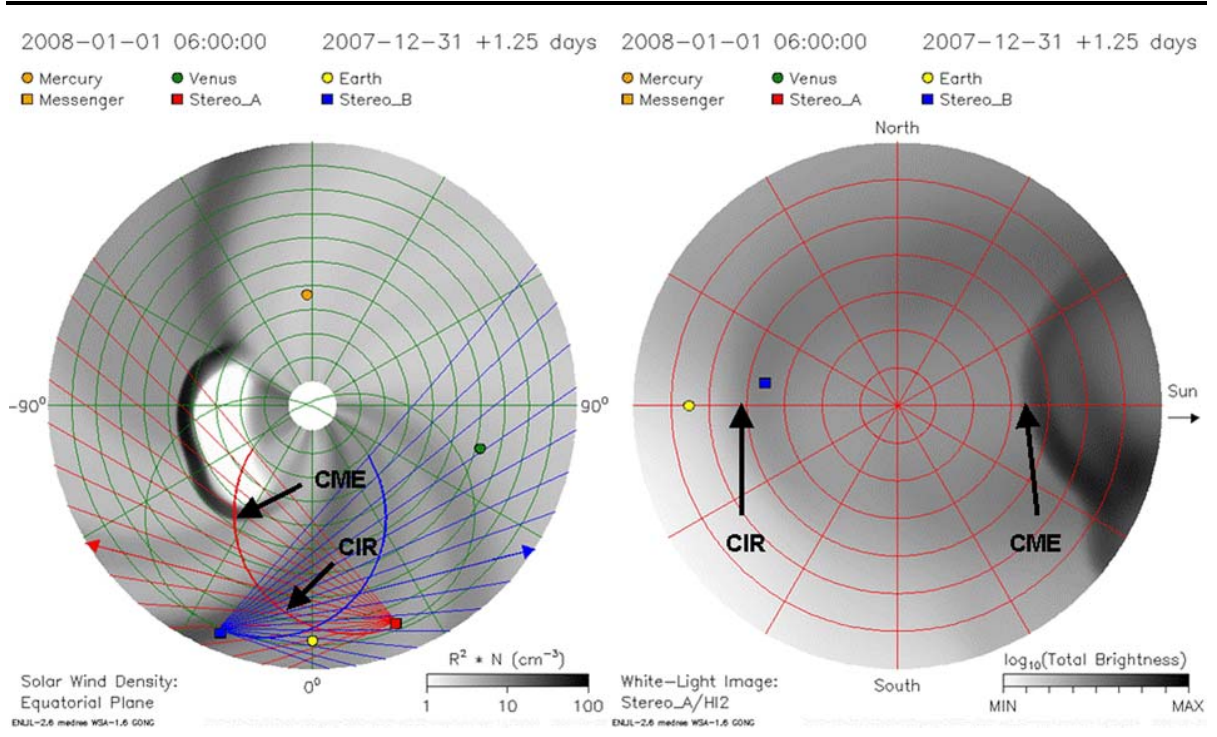

Figure 7 Heliospheric simulation of the 31 December 2007 CME shown on 1 January 2008 06:00 UT. Left panels show the normalized solar wind density on the equatorial plane between 0.1 and $1.1 \mathrm{AU}$. Right panel shows the synthetic white-light image as might be observed by STEREO HI-2A. Results are shown as in Figure 5.

by two ICMEs that merge into a single one. Note the curved leading edge of ICMEs and spots of enhanced densities caused by their interaction with the structured background solar wind.

The heliospheric disturbance caused by the 31 December 2007 east-limb CME is another example of a well-defined structure. Again, no ICME was detected by the in-situ instruments aboard heliospheric spacecraft. Figure 7 presents a simulated heliospheric disturbance caused by launching very large spherical homogeneous hydrodynamic ejecta similar to the ones considered so far. Note that the flank of the disturbance passes through Messenger, which was approaching Mercury (see Figure 7, left panel, orange box and sphere in the top-right sector). However, Messenger detected neither coronal ejecta nor interplanetary shock. Figure 8 presents results for the same event, but for the heliospheric disturbance initialized by launching the hydrodynamic ejecta with a flux-rope structure similar to the Krall and St. Cyr (2006) model. The location, direction, speed, and maximum angular extent were the same as for the cone model. The flux rope was inclined to the equatorial plane by $76^{\circ}$. This causes the disturbance to have a large latitudinal extent corresponding to coronagraph observations, but with a narrow longitudinal extent corresponding to Messenger measurements.

Multi-spacecraft remote and in-situ observations play a crucial role in constraining various models of ICMEs relevant for space weather prediction. Figures 9 and 10 show simulated heliospheric disturbances by the 26 April 2008 CME. The left panel of Figure 9 provides a global context and shows the ICME interacting with one present CIR. Additional visualizations can be made to better understand that three-dimensional dynamic interaction. The right panel of Figure 9 shows predicted temporal profiles at STEREO B, together with the observed values by the IMPACT and PLASTIC instruments. An interplanetary shock that was predicted to hit STEREO B on 29 April at 10 UT was observed a few hours later at 


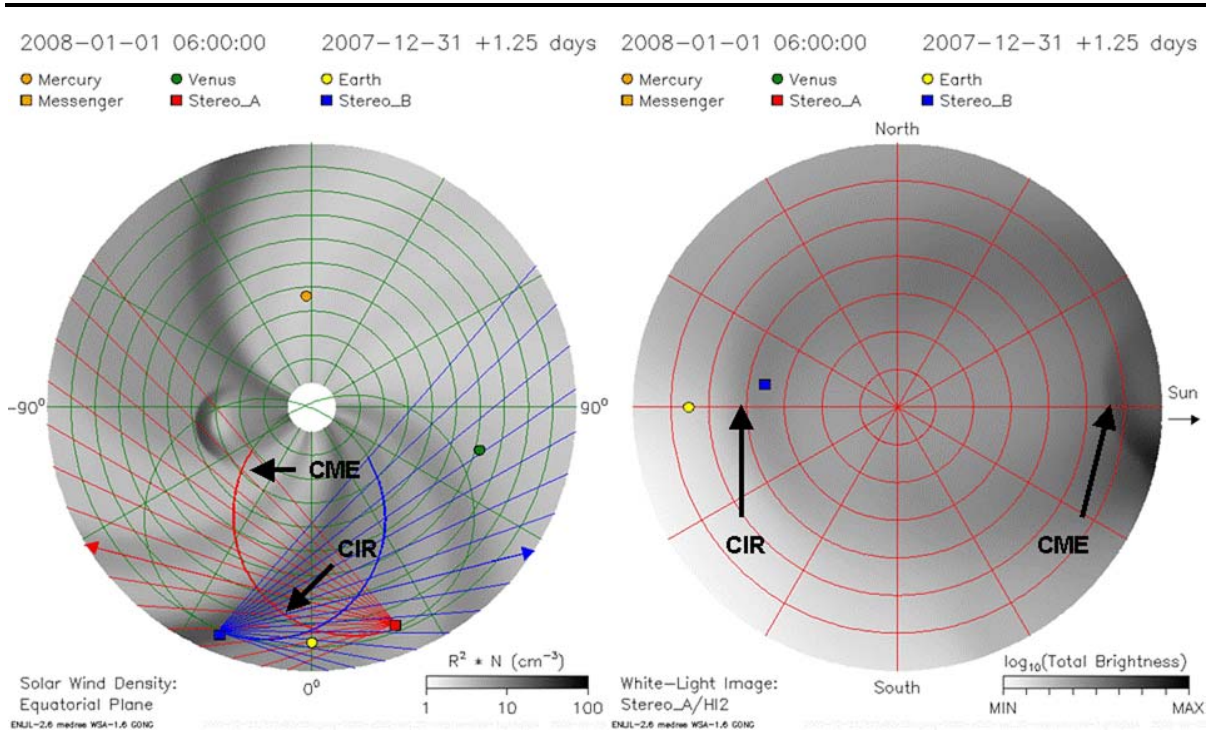

Figure 8 The same as in Figure 7, but for a simulation where the launched hydrodynamic ejecta has the geometric form of a flux rope (instead of hydrodynamic spherical cloud).

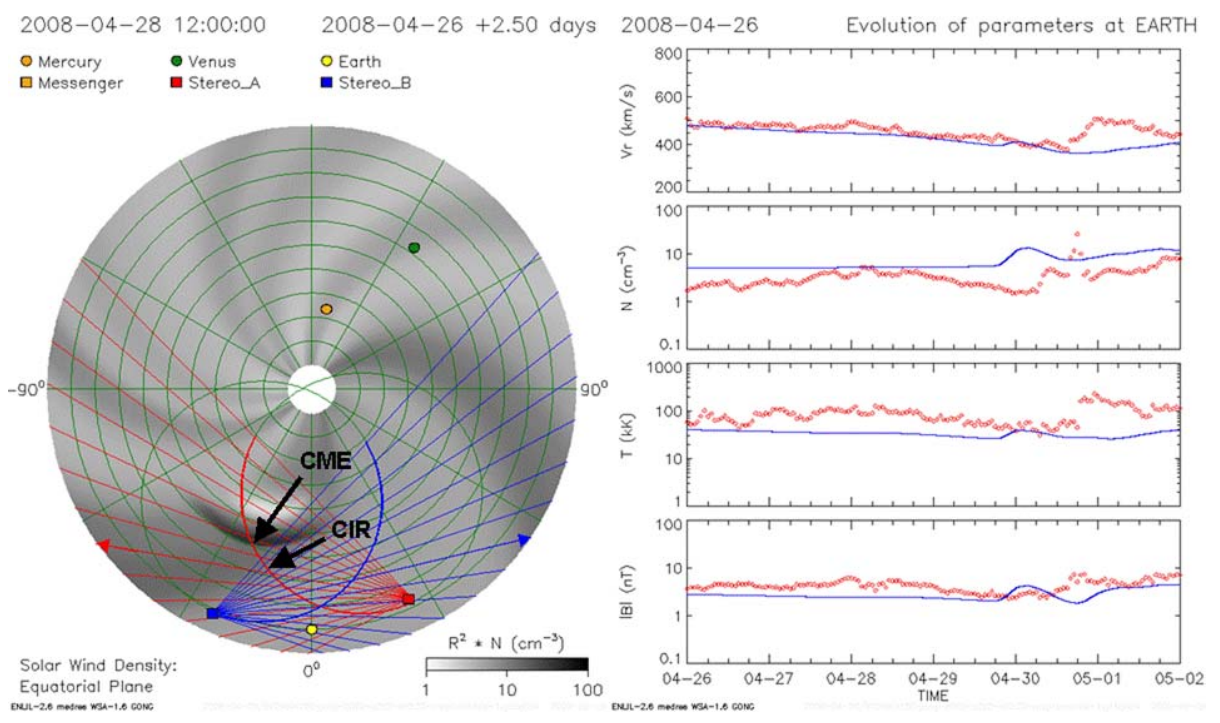

Figure 9 Heliospheric simulation of the 26 April 2008 CME. Left panel shows the normalized solar wind density on the equatorial plane between 0.1 and 1.1 AU on 28 April 2008 12:00 UT. Right panel shows the simulated (blue line) and observed (red dots) temporal profiles of the solar wind velocity, number density, mean temperature, and magnetic field strength (from top to bottom) at STEREO B.

14:15 UT. The panels in Figure 10 illustrate the asymmetric appearance of that interplanetary disturbance as might be seen by two imagers at different locations. Note that the leading edge of the ICME has a large dimple with enhanced intensity due to its interaction with the CIR. 


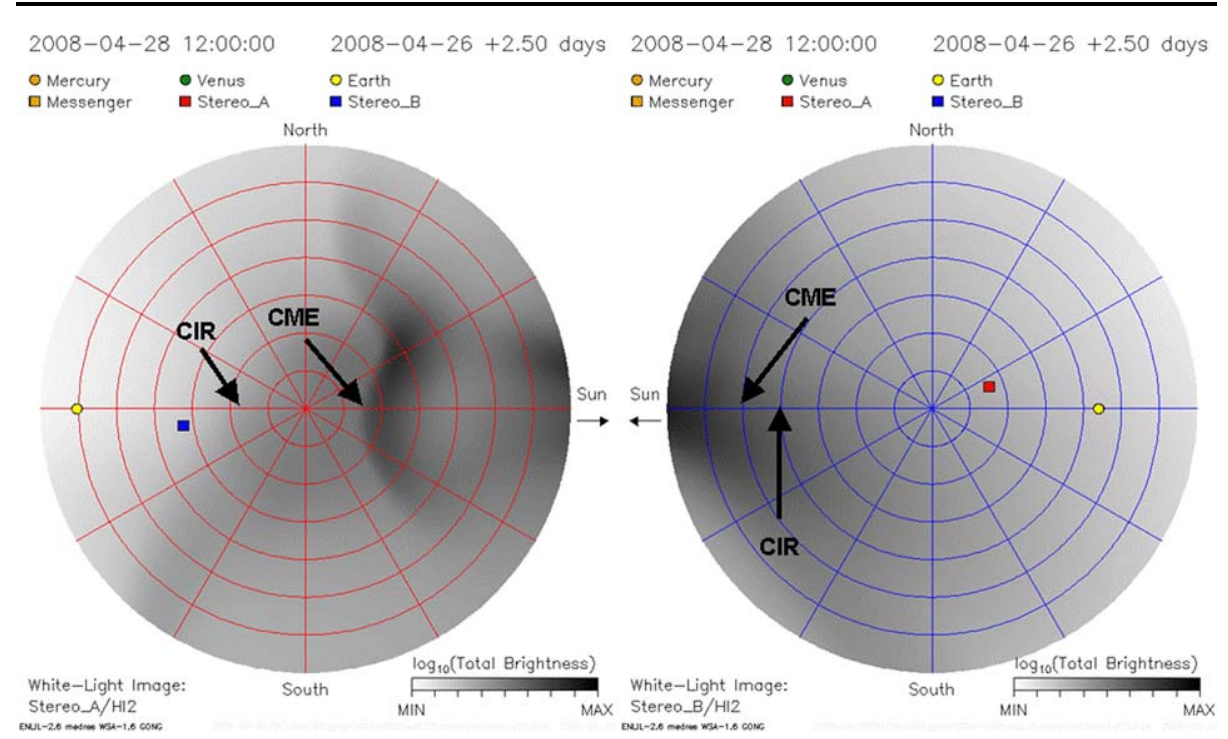

Figure 10 Synthetic white-light images as might be seen by STEREO HI-2A (left) and HI-2B (right) instruments on 28 April 2008 12:00 UT. Results are shown as described in Figure 5.

\section{Conclusions}

Interpretation of multi-spacecraft heliospheric observations and three-dimensional reconstruction of structured and evolving solar wind, as observed by heliospheric imagers (HI) onboard the STEREO spacecraft, is challenging. Numerical simulations can provide global context and hints of what can and cannot be observed. We develop a hybrid modeling system for simulation of corotating and transient heliospheric disturbances. Such simulations serve as a practical and efficient intermediate solution until better near-Sun observations and more sophisticated CME models become available. The main advantage of our approach is robust, fast, event-by-event simulation. Since CMEs expand as they propagate into the heliosphere, CMEs that are even one day apart can affect each other through the trailing rarefactions they create. This means that during the maxima of solar activity a continuous simulation of the heliosphere with all observed CMEs must be used to predict the effects on geospace. Such a requirement is beyond any current or foreseeable capabilities of self-consistent threedimensional MHD solar eruption models.

We use the heliospheric code ENLIL, simulate various scenarios of well-defined corotating solar wind streams and ejected transient density structures, and generate synthetic whitelight images at various locations. We illustrate that corotating interaction regions (CIRs) may as appear as transient structures in HI-2A but not in HI-2B. This asymmetry is due to a differing Thomson scattering response. Further, we illustrate that the same interplanetary coronal mass ejection (ICME) can show up with different white-light brightness depending on the observing position and that some ICMEs can eventually reach Earth without being detected by the STEREO HI-2 imagers. We also find that similar white-light intensity can be produced by different CME parts, and that similar CME parts can be observed with different white-light intensities. Finally, we provide an example of a practical application by presenting results for the 24 and 25 January 2007 CMEs. These two ICMEs interacted with each other and with the corotating stream structure. Real interplanetary CMEs can be even more complex, leading us to conclude that interpretation of STEREO/HI images requires 
iterative comparison with three-dimensional numeric simulations. By comparing the observations, we will be able to distinguish between the various runs to provide constraints. The presented visualizations will be incorporated at the NASA Community Coordinated Modeling Center (CCMC) to enhance the run-on-request and real-time heliospheric simulations. Collaboration between modelers and observers is necessary to understand three-dimensional heliospheric disturbances, interpret remote and in-situ observations, constrain ICME models, and develop space weather prediction capability.

Acknowledgements This work was supported by AFOSR/MURI, NASA/LWS, and NSF/CISM projects. We acknowledge data from Mount Wilson Observatory and Kitt Peak National Solar Observatory, ESANASA SOHO and STEREO spacecraft, and NASA National Space Science Data Center.

Open Access This article is distributed under the terms of the Creative Commons Attribution Noncommercial License which permits any noncommercial use, distribution, and reproduction in any medium, provided the original author(s) and source are credited.

\section{References}

Arge, C.N., Pizzo, V.J.: 2000, J. Geophys. Res. 105, $10465-10480$.

Arge, C.N., Odstrcil, D., Pizzo, V.J., Mayer, L.: 2003. In: Proc. Solar Wind 10, AIP Conf. Proc. 679, AIP, New York, $190-193$.

Case, A.W., Spence, H.E., Owens, M., Riley, P., Odstrcil, D.: 2008, Geophys. Res. Lett. 35, L15105. doi:10. 1029/2008GL034493.

Gopalswamy, N., Lara, A., Yashiro, S., Kaiser, M.L., Howard, R.A.: 2001, J. Geophys. Res. 106, $29207-$ 29218.

Harrison, R.A., Davis, C.J., Eyles, C.J., Bewsher, D., Crothers, S.R., Davies, J.A., Howard, R.A., Moses, D.J., Socker, D.G., Newmark, J.S., et al.: 2008, Solar Phys. 247, 171 - 193.

Krall, J., St. Cyr, O.C.: 2006, Astrophys. J. 652, 1740-1746.

Lee, C.O., Luhmann, J.G., Odstrcil, D., MacNeice, P., dePater, L., Riley, P., Arge, C.N.: 2008, Solar Phys. 254, 155 - 183. doi:10.1007/s11207-008-9280-y.

Michalek, G., Gopalswamy, N., Yashiro, S.: 2003, Astrophys. J. 584, $472-478$.

Odstrcil, D., Dryer, M., Smith, Z.: 1996, J. Geophys. Res. 101, $19973-19986$.

Odstrcil, D., Pizzo, V.J.: 1999a, J. Geophys. Res. 104, 483-492.

Odstrcil, D., Pizzo, V.J.: 1999b, J. Geophys. Res. 104, 28225 - 28239.

Odstrcil, D.: 2003, Adv. Space Sci. 32(4), 497-506.

Odstrcil, D., Riley, P., Zhao, X.P.: 2004, J. Geophys. Res. 109, A02116. doi:10.1029/2003JA010135.

Odstrcil, D., Pizzo, V.J., Arge, C.N.: 2005, J. Geophys. Res. 110, A02106. doi:10.1029/2004JA010745.

Owens, M., Arge, C.N., Spence, H.E., Pembroke, A.: 2005, J. Geophys. Res. 110, A12105.

Owens, M.J., Spence, H.E., McGregor, S., Hughes, W.J., Quinn, J.M., Arge, C.N., Riley, P., Linker, J., Odstrcil, D.: 2008, Space Weather, 6, S08001. doi:10.1029/2007SW000380.

Riley, P., Linker, J.A., Mikic, Z.: 2001, J. Geophys. Res. 100, 15889 - 15901.

Schwenn, R., dal Lago, A., Huttunen, E., Gonzalez, W.D.: 2005, Ann. Geophys. 23, 1033 - 1059.

Taktakishvili, A., Kuznetsova, M., MacNeice, P., Hesse, M., Rastaeter, L., Pulkinen, A., Chulaki, A.: 2009, Space Weather 7, S03004. doi:10.1029/2008SW000448.

Vourlidas, A., Howard, R.A.: 2006, Astrophys. J. 642, 1216-1221.

Webb, D.F., Howard, T.A., Fry, C.D., Kuchar, T.A., Odstrcil, D., Jackson, B.V., Bisi, M.M., Harrison, R.A., Morrill, J.S., Howard, R.A., Johnston, J.C.: 2009, Solar Phys. 256, 239-267. doi:10.1007s11207009-9351-8.

Xie, H., Ofman, L., Lawrence, G.: 2004, J. Geophys. Res. 109, CiteID A03109. doi:10.1029/2003JA010226.

Zhao, X.P., Plunkett, S.P., Liu, W.: 2002, J. Geophys. Res. 107, 1223. doi:10.1029/2001JA009143. 